\title{
Estimation of Micronutrients (Magnesium and Iron) in Periodontitis Patients with and without Type-2 Diabetes Mellitus
}

\author{
Pallavi Samatha Yalamanchili ${ }^{1}$, Nirupa Elisetti², Janaki Kesipeddy ${ }^{3}$, Bilwa Bindu A ${ }^{4}$, Bhagyasree Vegunta \\ 1, 2, 3, 4, 5 Department of Periodontics and Implantology, Drs. Sudha and Nageswara Rao \\ Siddhartha Institute of Dental Sciences, Chinnoutpally, Andhra Pradesh, India.
}

\section{ABSTRACT}

\section{BACKGROUND}

The focus of this study was to assess the relationship of nutrients (magnesium, iron) in periodontitis and diabetic patients. Serum magnesium $(\mathrm{Mg})$ and iron (Fe) levels were evaluated in periodontitis patients and were compared between diabetic patients with periodontitis and healthy controls.

\section{METHODS}

Total sixty subjects (18 females and 42 males) were recruited in this cross-sectional study and were divided into three groups - Group I (healthy subjects), Group II (chronic generalized periodontitis), Group III (Type II diabetes mellitus and chronic generalized periodontitis). Loe and Silness gingival index and probing pocket depth were used for categorizing chronic generalized periodontitis patients. Serum levels of magnesium and iron were estimated and then compared among the groups. Statistical analysis was done using one-way analysis of variance (ANOVA) and Newmann-Keuls multiple post-hoc procedures. Statistical significance was accepted for $\mathrm{P} \leq 0.05$.

\section{RESULTS}

Mean serum levels of magnesium ( $\mathrm{Mg}$ ) were 0.89 in Group 3 (Type II DM + CGP), 1.01 in Group 2 (CGP) against 1.52 in Group 1 (Control), which were statistically significant. Mean Iron (Fe) levels showed 165.56 in Group 3 (Type II DM + CGP), 137.77 in Group 2 (CGP) against 78.17 (Control) which is also statistically significant.

\section{CONCLUSIONS}

In this study, we found statistically significant decrease in magnesium and increase in serum iron levels in Group 3 (Type II DM + CGP) compared to Group 2 (CGP) and healthy controls. These findings suggest that trace elements also play an important role in the progression of periodontal disease.

\section{KEY WORDS}

Chronic Periodontitis, Diabetes Mellitus, Iron, Magnesium
Corresponding Author:

Dr. Nirupa Elisetti, Nirupa's Dental Zone Nallagandla, Hyderabad, Telangana - 500019, India

E-mail: nirupasdentalzone@gmail.com

DOI: $10.14260 / j e m d s / 2021 / 342$

How to Cite This Article:

Yalamanchili PS, Elisetti N, Kesipeddy J, et al. "Estimation of micronutrients (magnesium and iron) in periodontitis patients with and without type - 2 diabetes mellitus." J Evolution Med Dent Sci 2021;10(22):1645-1649, DOI: $10.14260 /$ jemds $/ 2021 / 342$

Submission 31-01-2021,

Peer Review 06-04-2021,

Acceptance 13-04-2021,

Published 31-05-2021.

Copyright (C) 2021 Pallavi Samatha Yalamanchili et al. This is an open access article distributed under Creative Commons Attribution License [Attribution 4.0 International (CC BY 4.0)] 


\section{BACKGROUND}

Periodontitis is an immune related disease which results from disruption of homeostasis between microorganisms and host related immune response and is influenced by several risk factors such as diabetes, smoking and diet. Balanced and sufficient diet helps in maintenance and functioning of different physiological and metabolic mechanisms happening inside the body. Research proves that inadequate diet is also associated with an increased risk of periodontal disease. ${ }^{1}$ Micronutrients like vitamins and minerals though required in minute quantities play a crucial role for functioning of biochemical reactions, immune responses, tissue regeneration and growth in our body. The most important enzymatic regulation in the body is also mediated by these micronutrients acting as co-factors for many enzymes. ${ }^{2}$

Diabetes mellitus (DM) is a chronic metabolic disease characterized by hyperglycaemia (increase in blood sugar) resulting from defective insulin secretion in the pancreas or due to insulin resistance which is decreased uptake of insulin at the cellular level. Insufficient minerals and increased oxidative stress in diabetic individuals further worsen the disease process by increasing insulin resistance at the cellular level, whilst chronic diabetes results in disruption of mineral homeostasis in the humans. ${ }^{3}$ Periodontitis is considered as sixth complication of diabetes mellitus [Loe H.et al 1993]. ${ }^{4}$ Both diabetes and periodontitis are chronic diseases and share common pathogenic mechanisms which is why they are considered to have a two-way relationship signifying worsened diabetic state in periodontitis patients and vice versa.

Magnesium is one such essential micro-nutrient that helps in eradication or elimination of free radicals that are generated inside the body and thereby reducing the oxidative stress. Both periodontitis and diabetes mellitus have a common culprit mechanism that disrupts the homeostasis of the host regulation which is none other than oxidative stress. ${ }^{5}$ Magnesium additionally helps in regulation of host immune response. (Mooren et al. 2013). Hypomagnesaemia results in increased neutrophil activation and functioning at the infected or inflamed sites whereas increased magnesium levels decrease the production of free radicals. (Bussiere et al 2002). ${ }^{6}$ The other micronutrient is iron which is a strong pro-oxidant in its free state resulting in increased generation of free radicals thereby increasing oxidative stress in the body by enzymatic catalytic reactions. Consequently, metallic iron is the reason for oxidative damage in diabetic individuals and plays a significant role in its progression. ${ }^{7}$ Accumulated reactive oxygen species in periodontal tissues progress the inflammatory reactions in periodontal tissues of diabetics. The objective of the study is to estimate and compare serum magnesium and iron levels in periodontitis patients with and without type-2 diabetes mellitus.

\section{METHODS}

This cross-sectional study included a total of sixty participants (18 females and 42 males) who visited the Department of Periodontics and Implantology from December 2017 to April 2018. The study protocol was approved by the institutional review board. Ethical clearance was also obtained from the same institution and a written informed consent was obtained from all the participants included in the study. The subjects were divided into three groups of twenty patients each. Group one was control, group two were systemically healthy with chronic generalized periodontitis diagnosed based on probing pocket depth of $>5 \mathrm{~mm}$ and group three were chronic generalized periodontitis diagnosed with type- 2 diabetes mellitus and under medication. Patients with known diabetes mellitus (type-2) for $>1$ year and under medication were included in the study. Patients with probing pocket depth of $\geq$ $5 \mathrm{~mm}$ for at least 30 percent of the sites were recognized as patients suffering from chronic generalized periodontitis and subjects with minimum compliment of 20 teeth in the oral cavity were selected for the study. Patients with presence of other systemic diseases that could influence the course of periodontal disease, smokers, pregnant and lactating women and patients who have undergone periodontal treatment for the past three months were excluded from the study. Overweight and obese individuals also were excluded from the study which could act as confounding factors. Individuals with previous history of mineral, vitamins and antioxidants intake were also not included in the study.

All the teeth present were first examined for presence or absence of gingival inflammation. Periodontal pockets were measured using William's periodontal probe. Venous blood sample (5 ml) was drawn from all the subjects using a disposable syringe under aseptic conditions. The blood was collected in a vial. A waiting period of a minimum of half an hour was required and then the collected blood was centrifuged at $2500 \mathrm{rpm}$ for 10 minutes and the supernatant serum was collected. Estimation of serum values of iron and magnesium were obtained from semi-automatic analyser using commercially available iron and magnesium kits [Coral diagnostic systems, Uttarakhand; India.]

\section{Statistical Analysis}

All the teeth present were examined for presence (or) absence of gingival inflammation and periodontal pockets. Pocket depth was measured at six sites per tooth. All the six measurements per tooth were added and a single value was obtained. All these single values per tooth were in-turn added to the number of teeth present and again a single value per individual was obtained for statistical analyses. The descriptive statistical value of mean was obtained even for magnesium and iron levels. Measure of variation, standard deviation was then calculated. One-way analysis of variance or ' $F$ ' test was measured to find out the significance of differences for more than two groups.

\section{RESULTS}

The data collected was analysed to determine the effect of destructive periodontal disease status on the serum levels of magnesium and iron. Comparison of magnesium among three groups showed that the mean value was 0.89 in Group III (CGP + Type II DM) which showed statistically decreased value compared to control (1.52) and Group II (CGP) of 1.01. [Table 1, Figure 1]. Comparison of iron also showed a statistical 
increase value in Group III (CGP + Type II DM) of 165.56 when compared to other groups of control (78.17) and Group II (137.77). [Table 1, Figure 2.] Pair-wise comparison of three groups with respect to magnesium scores by Newman-keuls multiple post-hoc procedures showed that between group I \& II, the P-value was 0.0101 which was statistically significant. On similar lines intergroup comparison between Group I \& III, the $P$ value was $P=0.0052$ which was statistically significant [Table 2].

Pair-wise comparison of three groups with respect to iron $(\mu \mathrm{g} / \mathrm{dl})$ scores by Newman-Keuls multiple post-hoc procedures showed a significant $\mathrm{P}$ value of $\mathrm{P}=0.0001$ between Group I \& Group III. Even between Group II \& Group III there was significant $P$ value of 0.0327 [Table 3].

\begin{tabular}{|c|c|c|c|c|c|}
\hline Variables & Groups & Mean & Standard Deviation & Minimum & Maximum \\
\hline \multirow{4}{*}{ Magnesium (mEq/L) } & Chronic generalized gingivitis & 1.52 & 0.90 & 0.14 & 3.50 \\
\hline & Chronic generalized periodontitis & 1.01 & 0.48 & 0.26 & 1.66 \\
\hline & Chronic periodontitis and diabetes mellitus & 0.89 & 0.37 & 0.27 & 1.71 \\
\hline & Total & 1.13 & 0.67 & 0.14 & 3.50 \\
\hline \multirow{4}{*}{ Iron $(\mu \mathrm{g} / \mathrm{dl})$} & Chronic generalized gingivitis & 78.17 & 34.06 & 18.82 & 139.40 \\
\hline & Chronic generalized periodontitis & 137.77 & 51.13 & 26.23 & 184.97 \\
\hline & Chronic periodontitis and diabetes mellitus & 165.56 & 35.46 & 41.25 & 201.00 \\
\hline & Total & 128.41 & 54.43 & 18.82 & 201.00 \\
\hline & $\begin{array}{l}\text { Table 1. Summary Statistics of M } \\
\text { Generalized Gingivitis, Chronic Gen }\end{array}$ & (mEq) & $\begin{array}{l}\text { ron }(\mu g d l) \text { in the Th } \\
\text { 'ronic Periodontitis }\end{array}$ & $\begin{array}{l}\text { oups } \\
\text { iabetes Me }\end{array}$ & \\
\hline
\end{tabular}

\begin{tabular}{|c|c|c|}
\hline Groups & \multicolumn{2}{|c|}{ Chronic Generalized GingivitisChronic Generalized PeriodontitisChronic Periodontitis and Diabetes Mellitus } \\
\hline Mean & \begin{tabular}{|l|l|}
1.52 & 1.01 \\
\end{tabular} & 0.89 \\
\hline SD & 0.90 & 0.37 \\
\hline Chronic generalized gingivitis & - & \\
\hline Chronic generalized periodontitis & $P=0.0101^{*}$ & \\
\hline \multicolumn{3}{|c|}{$\begin{array}{l}\text { Table 2. Pair Wise Comparison of the Three Groups (Chronic Generalized Gingivitis, Chronic Generalized Periodontitis, } \\
\text { Chronic Periodontitis with Diabetes Mellitus) with Respect to Magnesium (mEq/L) Scores by Newman-Keuls Multiple Post-Hoc Procedures }\end{array}$} \\
\hline
\end{tabular}

\begin{tabular}{|c|c|c|}
\hline Groups & Chronic Generalized Gingivitis Chronic Generalized Periodontitis & $\begin{array}{l}\text { Chronic Periodontitis and Diabetes } \\
\text { Mellitus }\end{array}$ \\
\hline Mean & 137.77 & 165.56 \\
\hline SD & 51.13 & 35.46 \\
\hline Chronic generalized gingivitis & - & \\
\hline Chronic periodontitis and diabetes mellitus & $\mathrm{P}=0.0001^{*}$ & - \\
\hline \multicolumn{3}{|c|}{$\begin{array}{l}\text { Table 3. Pair Wise Comparison of the Three Groups (Chronic Generalized Gingivitis, Chronic Generalized Periodontitis, } \\
\text { Chronic Periodontitis and Diabetes Mellitus) with Respect to Iron ( } \mu \mathrm{g} / \mathrm{dl} \text { ) Scores by Newman-Keuls Multiple Post-Hoc Procedures }\end{array}$} \\
\hline
\end{tabular}

\section{DISCUSSION}

Increasing evidence over the past few decades on the clinical and pathogenic relationship of periodontitis with systemic health has opened new perspectives of understanding the biochemical changes occurring in the human biological system. A very well-established ally exists between periodontal disease and diabetes with supporting molecular and cellular processes potentially linking these diseases. A collaborated authentication of various research by Taylor (1996, 2013) Mealey (2007), Pihlstrom B (2005), Chang (2012), Carstensen. M (2010), Aspriello (2011) potentiates a possible confluence between the two chronic inflammatory diseases, periodontitis, and diabetes. Periodontitis is one of the most common diseases occurring in the oral cavity. Research authenticates the relationship of diabetes with periodontitis with supporting mechanisms that signify the two-way relationship of the two diseases which cannot be denied either. According to our knowledge this is the first study known to compare the parameters of magnesium and iron in periodontitis patients and diabetic patients with periodontitis.

Categorization of subjects as chronic periodontitis was done by traditional diagnostic measures such as periodontal pocket depth with $\geq 30 \%$ of involved sites and Loe and Silness gingival index. Type II diabetics only were considered in the study while type I diabetics were not included to rule out genetic predisposition. Patients who were diagnosed with controlled type II diabetes at least for a year and under medication were included in the study as a certain amount of tissue destruction is required for understanding the mineral variability in the serum which are available in microgram and nanogram concentrations.

Furthermore, magnesium, a trace element plays an important role in mediating and controlling the oxidative stress in diabetic patients, a process which fails results in further exacerbating the disease process. ${ }^{8}$ Both periodontitis and diabetes which share a common pathogenic scenario of inception of free radicals and increased oxidative stress inside the body may lead to perturbation in homeostasis of trace elements and disturbing their individual mechanisms. Periodontitis, a risk factor for many systemic diseases which also include type-2 diabetes mellitus is also thought to have been associated with mineral deficiencies.

This cross-sectional study was designed to estimate and compare the association of two elements [Magnesium (Mg) and Iron (Fe)] in type II DM and chronic periodontitis patients against healthy controls. Type II diabetics only were considered in the study while type I diabetics were not included to rule out genetic predisposition. Patients who were diagnosed with type 2 diabetes at least for a year and under medication were included in the study as certain amount of tissue destruction is required for understanding the mineral 
variability in the serum which are available in microgram and nanogram concentrations. This requires a considerable lapse period for the patient to be in diabetic state.

Periodontitis is immune-inflammatory disease which results from disruption in homeostasis between microorganisms and host response with preloading of microorganisms and their inflammatory reaction which includes the increased number of primary defense cells of the body, the neutrophil. Further adding to this a decreased magnesium state (hypomagnesaemia) also results in premature activation of primary defense cells such as neutrophil resulting in hyper-inflammatory response leading to initiation and progression of disease. The results of our study showed decreased serum magnesium levels signifying a strong association between periodontitis and hypomagnesaemia. On the flip side high magnesium levels decreases the oxidative stress by inhibition of free radical production. ${ }^{6}$ Since both diabetes and periodontitis are related to increased oxidative stress in the body, hypomagnesaemia results in progression of both diseases correlating with the findings of our study with statistically significant decrease in serum magnesium levels in Group III [DM + CGP] against Group II [CGP] \& Group I [controls].

Albeit et al. ${ }^{6}$ explained the relationship between magnesium and periodontitis and stated that hypomagnesaemia aggravated inflammatory response to microbial load which increased the disease activity, and this is in accordance with our study wherein serum magnesium levels are less in Group II \& III compared to Group I.

Meisel et al. (2005) studied and concluded that magnesium supplementation helped improve periodontal health. Paolisso and Barbagallo (1995) stated that intracellular magnesium results in decreased tyrosine kinase activity and thereby increases insulin resistance further adding to aggravation of diabetic complications. Since, periodontitis is one of the complications of diabetes, hypomagnesaemia or decreased serum levels of magnesium are seen in Group III [CGP + Type II DM] compared to Group II [CGP] than in controls.

Research by Pushparani DS [2014] and Biju Thomas $[2016]^{9}$ also showed similar decrease in serum magnesium levels in [CGP + Type II DM] group compared to non-diabetic periodontitis subjects and controls coinciding with our results.

Iron always bounds to proteins such as transferrin, ferritin, lactoferrin and haemoglobin in the human beings. Iron in its free or metallic state is very dangerous and damaging to the body. E Rhodes et al. (2007) ${ }^{10}$ hypothesized that there would be an increase in iron concentration associated with haemoglobin leaking from vasodilated inflammatory pocket wall.

This is due to the association of increased expression of haem-binding proteins on the cellular surfaces of major periodontal pathogens such as P. gingivalis, T. denticola and $A$. actinomycetemcomitans which are most commonly present in periodontal pocket.

The aetiology behind initiation of inflammatory response in the sub-gingival environment of periodontal pocket is accumulation of microorganisms and generation of reactive oxygen species (ROS) such as hydrogen peroxide $\left(\mathrm{H}_{2} \mathrm{O}_{2}\right)$ and superoxide $\left(\mathrm{O}_{2}^{-}\right)$.
Iron in its free state catalyses the formation of toxic free radicals from $\left(\mathrm{H}_{2} \mathrm{O}_{2}\right) .{ }^{11}$ As periodontitis and diabetes are both chronic inflammatory diseases with increased free radical formation, increased free iron acts as a pro-oxidant increasing the oxidative stress of the body and aggravating destruction. This increase in serum iron (Fe) levels is seen in our study especially in Group III compared to Group II \& I.

The redox active metal ions $(\mathrm{Cu} 2+$ and $\mathrm{Fe} 3+)$ have been implicated in catalysing the auto-oxidation of glycoaldehyde and generation of hydroxyl radical leading to production of glyoxal and associated $\alpha$-oxoaldehyde derived advanced glycation end products (AGEs) formation. A wealth of experimental evidence supports the hypothesis that AGEs formed from glyoxal, methylglyoxal and 3-deoxy glucosone have an etiological role in development of diabetic complications and other diseases.

This study is a cross-sectional designed study with a small sample size where in, the relationship between nutrient status and periodontitis cannot be accurately established is a drawback. The duration of diabetic state of the participants in the study was not considered, which correlates to the amount of destruction with increased time the patient has suffered from the disease. This again is a drawback of the study. Further established research with increased sample size and with consideration of various confounding factors that could also act as variables authenticates the correlation between periodontitis and nutrients especially in diabetic patients.

\section{CONCLUSIONS}

From the above study, we can conclude that there was statistical decrease in serum magnesium levels and increase in serum iron levels in the group with both diabetes and periodontitis compared to periodontitis and healthy controls. Further longitudinal studies with larger sample size should be carried out to ascertain the association between nutrition and periodontitis.

Data sharing statement provided by the authors is available with the full text of this article at jemds.com.

Financial or other competing interests: None.

Disclosure forms provided by the authors are available with the full text of this article at jemds.com.

\section{REFERENCES}

[1] Van Dyke TE, Sheilesh D. Risk factors for periodontitis. ] Int Acad Periodontol 2005;7(1):3-7.

[2] Staudte H, Kranz S, Völpel A, et al. Comparison of nutrient intake between patients with periodontitis and healthy subjects. Quintessence Int 2012;43(10):907-16.

[3] Saha-Roy S, Pal S, Bera S, et al. Status of serum magnesium, zinc \& copper in patients suffering from type2 diabetes mellitus. Journal of Drug Delivery and Therapeutics 2014;4(1):70-2.

[4] Löe H. Periodontal disease. The sixth complication of diabetes mellitus. Diabetes Care 1993;16(1):329-34. 
[5] Chapple IL, Matthews JB. The role of reactive oxygen and antioxidant species in periodontal tissue destruction. Periodontol 2000 2007;43:160-232.

[6] Meisel P, Schwahn C, Luedemann J, et al. Magnesium deficiency is associated with periodontal disease. J Dent Res 2005;84(10):937-41.

[7] Pushparani DS. Serum zinc and iron level in type 2 diabetes mellitus with periodontitis. Int J Pharm Tech Res 2015;7(1):165-71.

[8] Pushparani DS, Anandan SN, Theagarayan P. Serum zinc and magnesium concentrations in type 2 diabetes mellitus with periodontitis. J Indian Soc Periodontol 2014;18(2):187-93.
[9] Shetty A, Bhandary R, Thomas B, et al. A comparative evaluation of serum magnesium in diabetes mellitus type 2 patients with and without periodontitis- a clinicobiochemical study. J Clin Diagn Res 2016;10(12):ZC59-61.

[10] Rhodes ER, Menke S, Shoemaker C, et al. Iron acquisition in the dental pathogen actinobacillus actinomycetemcomitans: what does it use as a source and how does it get this essential metal? Biometals 2007;20(3-4):365-77.

[11] Pushparani DS, Nirmala S. High level of serum calcium and iron influences the risk of type 2 diabetes mellitus with periodontitis. Journal of Asian Scientific Research 2014;4(2):70-82. 